\title{
Entornos Virtuales para el Diseño de Producto en el Espacio Público
}

Mónica Val Fiel ${ }^{\mathrm{a}}$,

aDepartamento de Expresión Gráfica Arquitectónica, Escuela Técnica Superior de Ingeniería del Diseño, Universitat Politècnica de València, movalfie@ega.upv.es

\begin{abstract}
The subject 'Design and Public Space', taught in the Master's Degree in Design Engineering of the UPV, presents the design as a strategy of intervention in the public space of the city. The course explores the possibilities offered by new technologies in the field of design, transferring the visualization of the project developed during the course to virtual environments.
\end{abstract}

Immersive virtual reality environments incorporates experiential perception to the project development, and in this context, the tool transcends its technological incentive and instrumental role, and also stands out as an innovative means and a potential communication tool.

Virtual Reality as a tool for the development of design project, enables to capture the attention of the students, improve the learning of specific contents of the subject, and promote the use of innovative tools in the field of design.

Keywords: Virtual Reality, CAVE, Immersive Interaction, Products Design, Public Space.

\section{Resumen}

La asignatura 'Diseño y Espacio Público', que se imparte dentro del Máster Universitario en Ingeniería del Diseño de la UPV, presenta el diseño como estrategia de intervención en el espacio público de la ciudad. La asignatura explora las posibilidades que ofrecen las nuevas tecnologías en el ámbito del diseño, trasladando la visualización del proyecto que se desarrolla durante el curso a entornos virtuales.

Los entornos inmersivos de Realidad Virtual incorporan la experimentación vivencial al desarrollo del proyecto y en este contexto, la herramienta trasciende su aliciente tecnológico y papel instrumental, destacándose tanto como un medio innovador, como una potencial herramienta de comunicación. 
La Realidad Virtual como herramienta para el desarrollo del proyecto de diseño, permite captar la atención del alumno, mejorar el aprendizaje de contenidos específicos de la asignatura, y promover el uso de herramientas innovadoras dentro del campo del diseño.

Palabras clave: Realidad Virtual, CAVE, Interacción Inmersiva, Diseño de producto, Espacio Público.

\section{Introducción}

La asignatura 'Diseño y Espacio Público', antes 'Diseño de productos para Espacios Públicos y Colectividades 1', se imparte dentro del bloque de Productos de Uso Colectivo en el seno del Máster Universitario en Ingeniería del Diseño de la Universitat Politècnica de València. Desde su primera edición en 2008, la signatura está orientada al ámbito de la colectividad y, a través de una visión pluridisciplinar, presenta el diseño como estrategia de intervención en el espacio público de la ciudad.

La asignatura aborda de forma teórica, metodológica e instrumental el proyecto de diseño en el espacio público: lugares, actividades, signos y señales, intangibles y efímeros, etc. Cada año, la asignatura plantea el diseño y la elaboración de un proyecto, que se inserta en el espacio público y que se desarrolla a lo largo de un semestre del curso. En los dos últimos años, la asignatura ha planteado un proyecto transversal junto con la asignatura de 'Diseño de instalaciones temporales y espacios expositivos' perteneciente al mismo bloque, centrando el objeto de proyecto en una instalación temporal que bajo determinados condicionantes se integra en el espacio público urbano.

Desde su primera edición, la asignatura explora las posibilidades que ofrecen las nuevas tecnologías en el ámbito del diseño, y en este caso, traslada la visualización del proyecto a entornos virtuales, introduciendo una herramienta innovadora dentro del proceso de diseño. El alumno, en un determinado estadio de desarrollo del proyecto, se sumerge en sus propias ideas. Los entornos virtuales acercan el proyecto a una realidad física pero intangible, siendo de gran utilidad para su desarrollo, permitiendo desde una perspectiva más amplia, validar las ideas y efectuar ajustes de escala, forma o materiales.

William Winn, en relación a este argumento y en su vinculación con aplicaciones educativas, justifica que "Los procesos psicológicos que se activan en la realidad virtual inmersiva son muy similares a los procesos psicológicos que operan cuando las personas construyen el conocimiento mediante la interacción con los objetos y acontecimientos en el mundo real". 


\section{Antecedentes}

Este artículo presenta la Realidad Virtual Inmersiva como una tecnología gráfica de visualización que introduce un desarrollo innovador en el proyecto de diseño. Las nuevas tecnologías han rediseñado los procesos de diseño y han ampliado el panorama de posibilidades en la implementación de nuevas herramientas para su desarrollo.

En una primera fase, el dibujo es considerado la principal herramienta para el desarrollo del proyecto. En fases sucesivas, el uso del Diseño Asistido por Ordenador complementa y multiplica la exploración de sus procesos. Algunos programas de diseño asistido incorporan además la fase de análisis, optimización y evolución del proyecto, correspondientes a la ingeniería asistida por ordenador, o permiten completar el ciclo de desarrollo del producto, con el control sobre la gestión de la fabricación del producto, que se correspondería con la última fase del proceso.

Sin embargo, en todo este proceso de diseño, en el que las herramientas gráficas desempeñan un importante papel, los aspectos perceptivos o de integración en el ámbito de actuación quedan reducidos a fotomontajes o infografías, en los que se hace coexistir, por superposición de manera estática, el proyecto desarrollado con fotografías reales o reproducciones modeladas del espacio en el que se integra el proyecto.

La relación de la instalación con el usuario y la integración de la instalación en el entorno urbano son contenidos específicos de la asignatura 'Diseño y Espacio Público'. Por ello, es fundamental reforzar los aspectos perceptivos de interacción del usuario con la instalación e integración con el ámbito de actuación propuesto, es una de las cuestiones clave. Uno de los retos importantes al que se enfrentan los alumnos es hacer coexistir las dos escalas de actuación que intervienen en el desarrollo del proyecto: la escala espacial del emplazamiento y la escala objetual del producto.
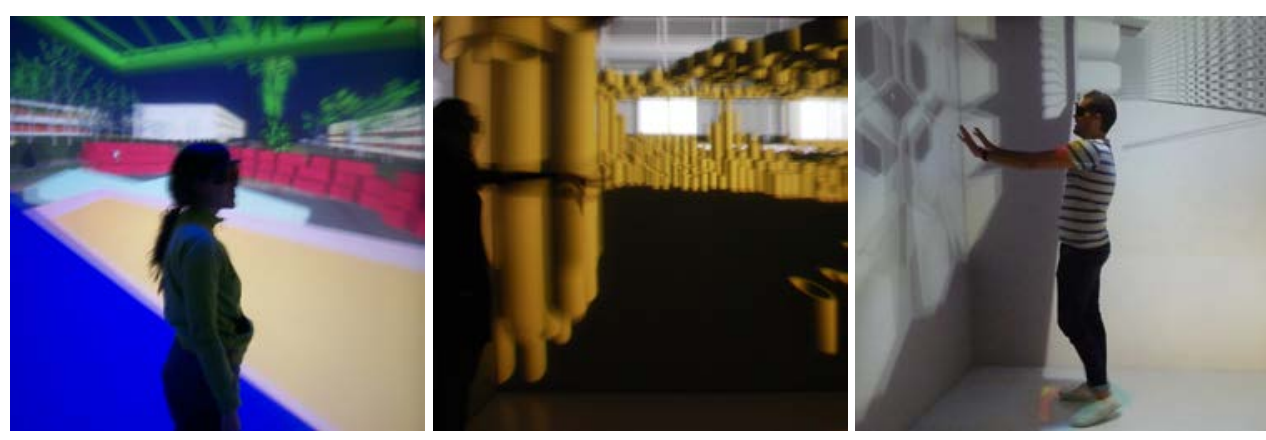

Fig. 1 Alumnos interaccionando con sus proyectos (de izquierda a derecha): Ana Lucía Zuluaga (curso 20082009), Carolina Gómez (curso 2010-2011) y Francisco Moreno (curso 2013-2014). 


\section{Objetivos}

El objetivo es incorporar en el desarrollo de proyecto la Realidad Virtual (RV) como herramienta del proceso, que permita reforzar los aspectos perceptivos y verificar algunos de los condicionantes intrínsecos al diseño. Con ello se plantea:

- Promover el uso de herramientas innovadoras. Utilizar la RV como herramienta para el desarrollo del proyecto, tan aceptado dentro de nuestra cultura visual en contextos como el del cine y los videojuegos, y que permite captar la atención del alumno, que se muestra interesado y motivado ante la propuesta.

- Contribuir a la mejora docente, abordando desde un amplio espectro dos de los contenidos específicos de la asignatura 'Diseño y Espacio Público'. El uso de esta herramienta multiplica exponencialmente la interacción del alumno con el proyecto, y hace visible su integración con el ámbito propuesto.

\section{Desarrollo de la innovación}

En una fase intermedia del desarrollo del proyecto, se propone trasladar la dimensión de visualización del papel o la pantalla a un espacio virtual, y obtener una nueva percepción simulada del mismo a través de una experiencia inmersiva.

Dentro del proceso iterativo que incluye el desarrollo proyectual: elaboración de alternativas, selección, constante verificación de las ideas, etc., la RV se presenta como una herramienta más, que se implementa en un determinado estadio del desarrollo del proyecto, junto con el uso del resto de herramientas de diseño asistido por ordenador más consolidadas.

Los denominados métodos inmersivos permiten, con dispositivos de localización y posicionamiento, restituir en tiempo real la visualización del proyecto junto con el entorno en función de los movimientos del usuario, que se desplaza así en un escenario construido. De este modo se promueve la experimentación del proyecto como una realidad espacial física pero intangible, proporcionando un nuevo nivel de interacción con las ideas propuestas por el alumno y con la forma en que estas se disponen en el espacio propuesto.

Las instalaciones del Visionarium de la Universitat Politècnica de València dependen del Área de Sistemas de Información y Comunicaciones (ASIC), y se encuentran situadas en la Ciudad de la Innovación (http://visionarium.blogs.upv.es). 
Es importante señalar que el desarrollo de la actividad potencia:

- La participación activa por parte del alumno.

- La experiencia directa en primera persona por parte del alumno, en un proceso interactivo que desarrolla a su ritmo.

- La comunicación eficaz de las ideas por parte del alumno que se sumerge en su proyecto.
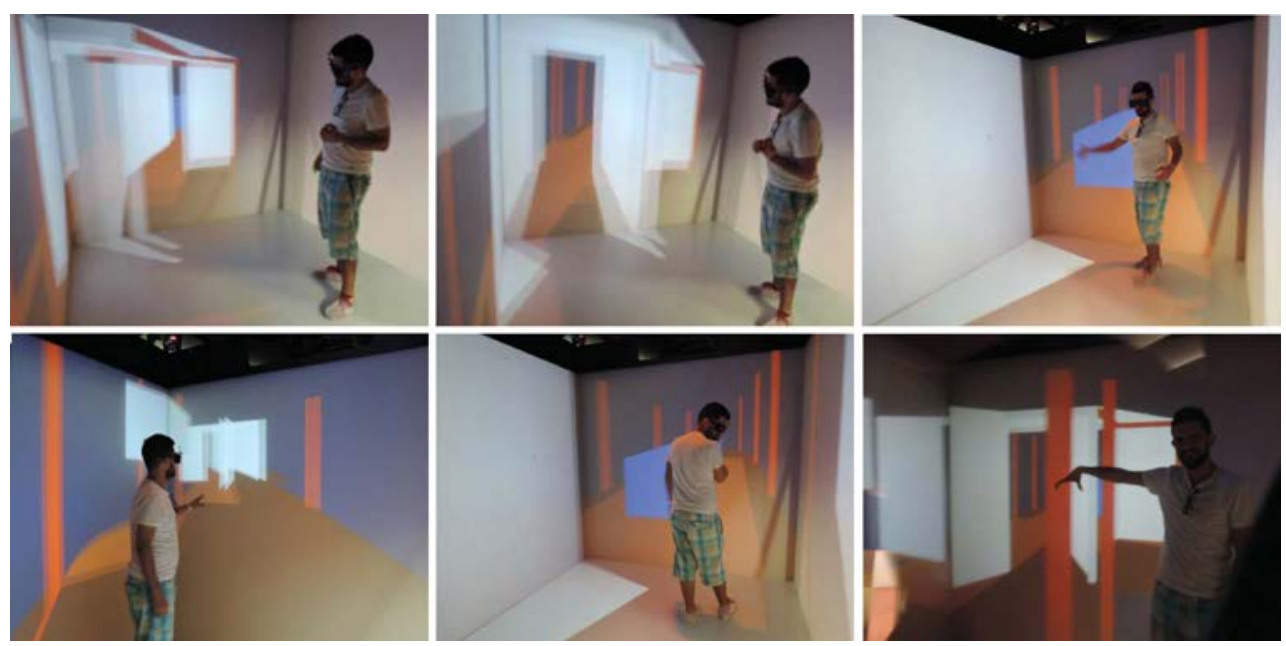

Fig. 2 Interacción del alumno Antonio Cobaleda Cordero con su proyecto. Curso 2014-2015

El usuario interactúa en la CAVE, un cubo de 2,5x2,5x2,35 m., con unas gafas con visión estereoscópica, que le permiten restituir de manera tridimensional el proyecto. Las gafas incorporan unos sensores de localización, que determinan la posición del usuario y refrescan en las cuatro pantallas de proyección de la CAVE cualquier movimiento en tiempo real. El entorno existente fuera de las dimensiones del cubo se proyecta e integra con el espacio interior, manteniendo en todo momento la vinculación con el ámbito de actuación.

En el desarrollo de la actividad, la proyección en las cuatro pantallas de la CAVE responde a dos niveles de decisión por parte del alumno. En un primer nivel, el alumno determina qué parte de la instalación proyectada incluye en el área de interacción de la CAVE y prepara los ficheros de intercambio para ello. En un segundo nivel, su posición y movimiento son los que determinan lo que se proyecta en las pantallas de la CAVE. Es el usuario/alumno el que elige el punto de vista, cuya representación se actualiza en tiempo real y de este modo es compartida con el resto del grupo.

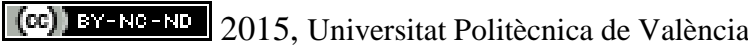

Congreso In-Red (2015) 

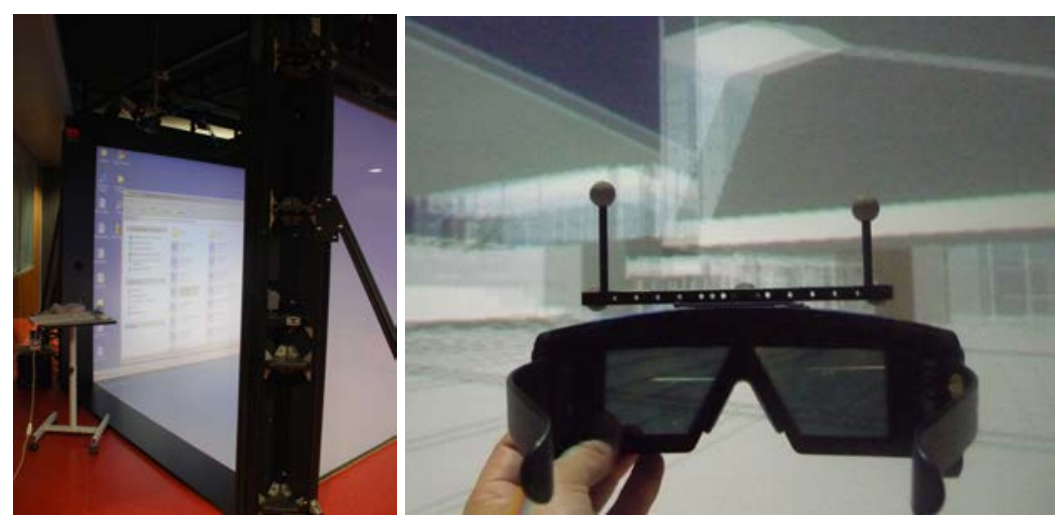

Fig. 3 Izquierda: CAVE Cave Automatic Virtual Environment. Derecha: gafas de visión estereoscópica con localizadores de posición

\section{Resultados}

Con el fin de evaluar los resultados de la implementación de la innovación tecnológica, en las dos últimas ediciones del máster (curso 2013-14 y 2014-15) se ha pasado un cuestionario al alumnado que ha desarrollado la actividad. Puesto que los condicionantes han sido los mismos en las dos ediciones, y dado el número reducido de alumnos en los grupos, se ha considerado la suma de ambas ediciones.

En este caso, se trata de una muestra de conveniencia, no probabilística, puesto que utiliza el conjunto del alumnado de la clase. La población que ha utilizado la tecnología en estas dos ediciones ha sido de 21 alumnos, y el muestreo que se obtuvo fue de 20 , de manera que puede considerarse representativa del conjunto de la población. En este caso el error muestral fue de 4,5\%, considerando la situación más desfavorable en el análisis estadístico, obteniendo así un nivel de confianza del 95\%.

La encuesta cumplimentada por el alumnado se ha centrado en dos aspectos. Por una parte, una valoración de los contenidos específicos que ofrece la tecnología implementada en el marco del proyecto de diseño, y por otra, con datos vinculados exclusivamente a la tecnología. Agrupando todas aquellas cuestiones que afectan a los contenidos específicos del proyecto, se han destacado las relacionadas con:

- Adecuación del uso de la herramienta a la fase del desarrollo del proyecto.

- Descripción del uso de la tecnología.

- Aspectos más relevantes del uso de la herramienta.

- Adecuación de los objetivos al uso de la herramienta. 
Desde la primera edición del máster y a lo largo de las sucesivas, se ha consolidado la implementación de la tecnología en una fase intermedia de desarrollo del proyecto. De los datos obtenidos en las dos últimas ediciones, el 75\% de los encuestados reafirmaron su uso idóneo en esta fase, en la que el proyecto se encuentra en un nivel medio de evolución, frente a un 5\% que proponía su implementación en una fase inicial y el último 20\% que lo desplazaba a una fase final del desarrollo.

Tras la implementación de la tecnología, la valoración de los alumnos ha destacado su uso como: interesante (80\%), útil (70\%), innovador (40\%), estimulante (40\%) y complicado (20\%).

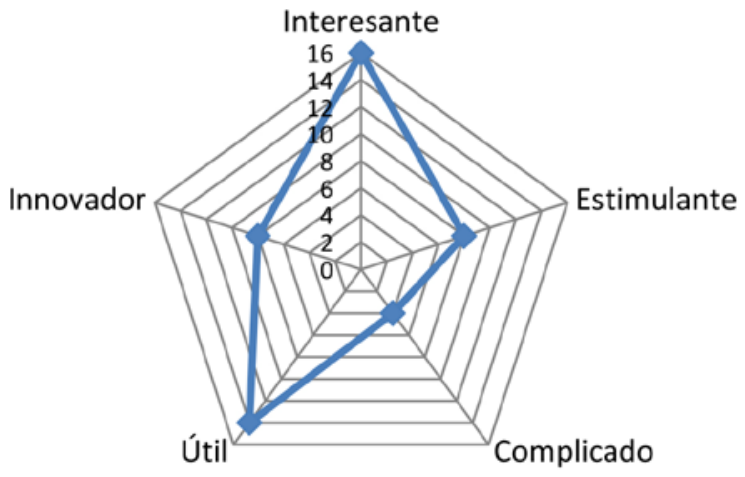

Fig. 4 Descripción del uso de la tecnología. Resultados de las ediciones 2013-2015

Tabla 1. Resultados del análisis de frecuencias de los descriptores del uso de la tecnología

\begin{tabular}{l|l|c|c|c}
\hline \multicolumn{2}{c|}{} & \multicolumn{2}{c}{ Respuestas } & \multirow{2}{*}{$\begin{array}{c}\text { Porcentaje } \\
\text { de Casos }\end{array}$} \\
\cline { 3 - 4 } \multicolumn{2}{c|}{} & $\mathrm{N}$ & Porcentaje & \\
\hline \multirow{2}{*}{ Descripción de la tecnología ${ }^{\mathrm{a}}$} & Interesante & 16 & $32,0 \%$ & $80,0 \%$ \\
\cline { 2 - 5 } & Complicado & 4 & $8,0 \%$ & $20,0 \%$ \\
\cline { 2 - 5 } & Útil & 14 & $28,0 \%$ & $70,0 \%$ \\
\cline { 2 - 5 } & Estimulante & 8 & $16,0 \%$ & $40,0 \%$ \\
\cline { 2 - 5 } & Innovador & 8 & $16,0 \%$ & $40,0 \%$ \\
\hline Total & & 50 & $100,0 \%$ & $250,0 \%$ \\
\hline
\end{tabular}

2 ¿Cómo describirías el uso de la tecnología?

\section{(cc) BY-NC-ND 2015, Universitat Politècnica de València}

Congreso In-Red (2015) 
En relación a los aspectos más relevantes que son trabajados con el uso de la herramienta, la valoración de los alumnos destacó que permite: una visualización a escala y en contexto (75\%), una experiencia dinámica (60\%), facilita la comunicación (40\%), la interacción en tiempo real (40\%), la motivación (20\%) y su facilidad de uso (10\%).

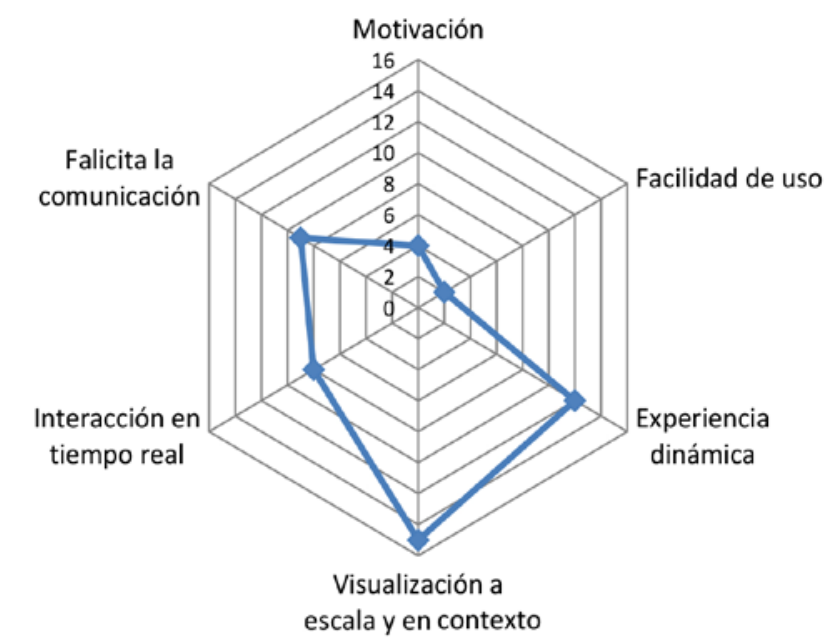

Fig. 5 Aspectos más relevantes del uso de la herramienta. Resultados de las ediciones 2013-2015

Tabla 2. Resultados del análisis de frecuencias de los aspectos relevantes del uso de la tecnología

\begin{tabular}{|c|c|c|c|c|}
\hline & & \multicolumn{2}{|c|}{ Respuestas } & \multirow{2}{*}{$\begin{array}{c}\text { Porcentaje } \\
\text { de Casos }\end{array}$} \\
\hline & & $\mathrm{N}$ & Porcentaje & \\
\hline \multirow{6}{*}{$\begin{array}{l}\text { Aspectos } \\
\text { relevantes del } \\
\text { uso }^{3}\end{array}$} & Motivación & 4 & $8,0 \%$ & $20,0 \%$ \\
\hline & Experiencia dinámica & 12 & $24,0 \%$ & $60,0 \%$ \\
\hline & Interacción en tiempo real & 8 & $16,0 \%$ & $40,0 \%$ \\
\hline & Facilidad de uso & 2 & $4,0 \%$ & $10,0 \%$ \\
\hline & Visualización a escala / en contexto & 15 & $30,0 \%$ & $75,0 \%$ \\
\hline & Facilita la comunicación & 9 & $18,0 \%$ & $45,0 \%$ \\
\hline \multicolumn{2}{|l|}{ Total } & 50 & $100,0 \%$ & $250,0 \%$ \\
\hline
\end{tabular}

3 Destaca los aspectos más relevantes del uso de la herramienta (Interacción en la CAVE) 
La herramienta permite un uso adecuado tanto para el diseño de productos como para el acondicionamiento de espacios, siendo una de las cuestiones que la asignatura hace coexistir. Además, se destaca como adecuada tanto para validar la idea como su escala de intervención e integración en el contexto. La valoración de los alumnos destacó el uso de la herramienta como adecuado según los siguientes resultados: la visualización del proyecto a escala y en su contexto fue uno de los aspecto valorado por el $80 \%$ de las respuestas, el acondicionamiento de los espacios fue destacado por el $60 \%$, siendo un $60 \%$ las respuestas que destacan la herramienta para validad la idea del proyecto y un $45 \%$ el diseño exclusivo del producto.

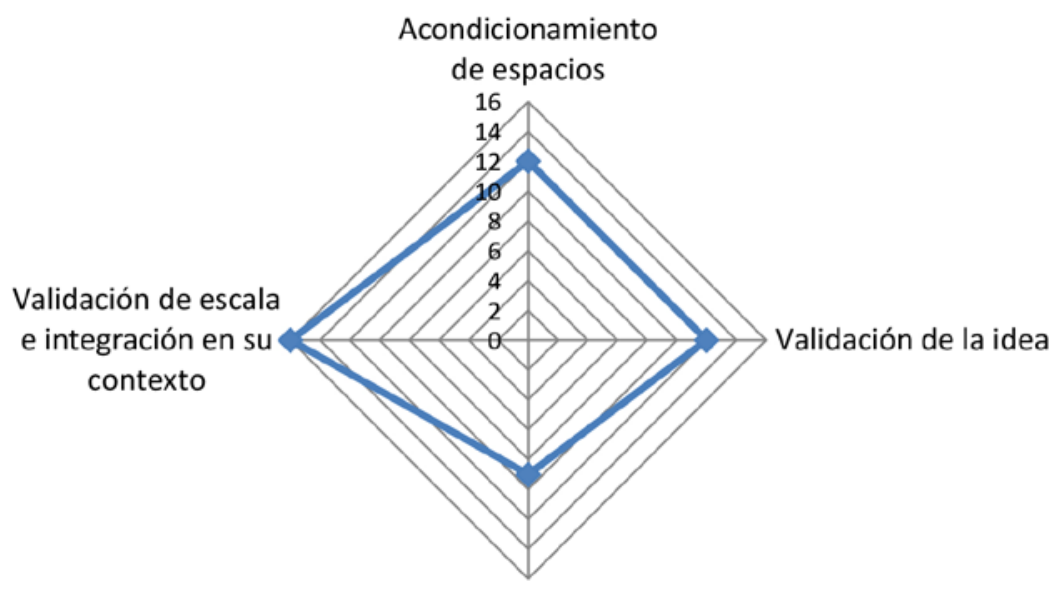

Diseño de producto

Fig. 6 Adecuación de los objetivos al uso de la herramienta. Resultados de las ediciones 2013-2015

Tabla 3. Resultados del análisis de frecuencias de la adecuación del uso de la tecnología

\begin{tabular}{l|l|c|c|c}
\hline \multicolumn{2}{c|}{} & \multicolumn{2}{|c|}{ Respuestas } & $\begin{array}{c}\text { Porcentaje } \\
\text { de Casos }\end{array}$ \\
\cline { 3 - 4 } \multicolumn{2}{c|}{} & $\mathrm{N}$ & Porcentaje & \\
\hline \multirow{2}{*}{ Adecuación del uso 9} & Acondicionamiento de espacios & 12 & $24,5 \%$ & $60,0 \%$ \\
\cline { 2 - 4 } & Diseño de producto & 9 & $18,4 \%$ & $45,0 \%$ \\
\cline { 2 - 4 } & $\begin{array}{l}\text { Validación de la escala e integración } \\
\text { en su contexto }\end{array}$ & 16 & $32,7 \%$ & $80,0 \%$ \\
\cline { 2 - 4 } & Validación de la idea & 12 & $24,5 \%$ & $60,0 \%$ \\
\hline Total & 49 & $100,0 \%$ & $245,0 \%$ \\
\hline
\end{tabular}

9 ¿Para qué consideras más adecuado el uso de la herramienta CAVE?

\section{(cc)) EY-NC-ND 2015, Universitat Politècnica de València}

Congreso In-Red (2015) 
La mayor parte de las valoraciones en relación exclusiva a la tecnología (escala Likert), sin entrar en las especificidades de los contenidos de la asignatura, se sitúa en los últimos niveles de conformidad.
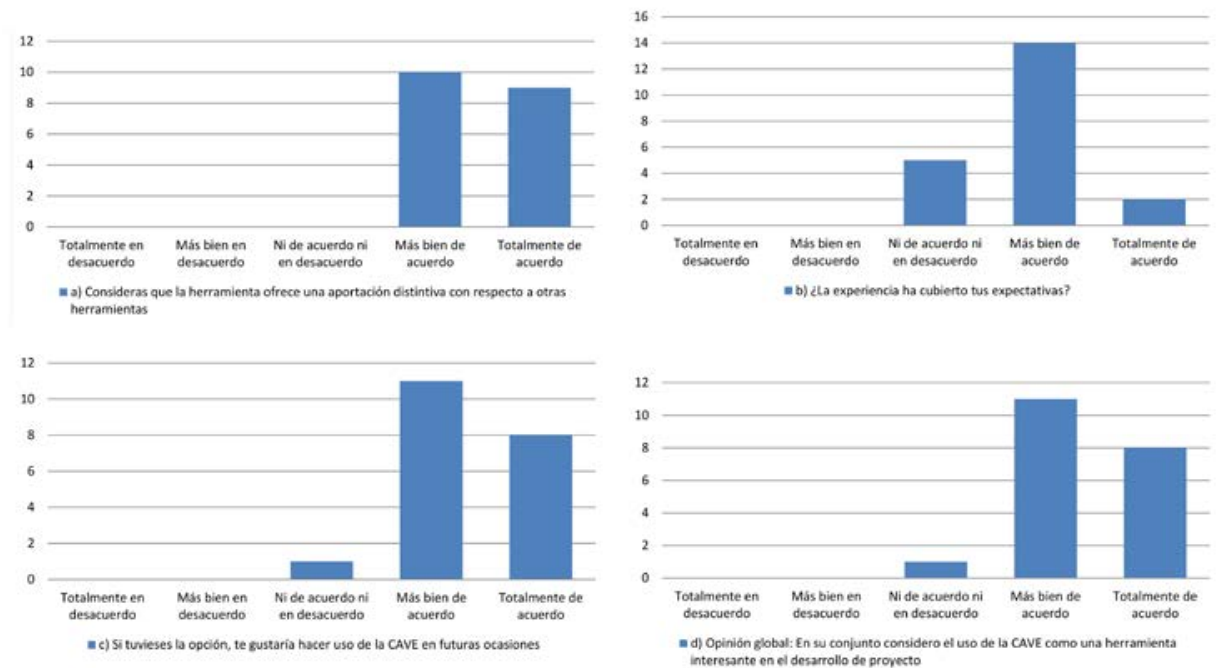

Fig. 7 Resultados de las ediciones 2013-2015. Valoración de la tecnología

En la encuesta propuesta en las últimas dos ediciones, los alumnos afirmaron que el uso de la RV es una herramienta interesante para el desarrollo del proyecto [punto d) fig. 7], el 40\% están totalmente de acuerdo, seguido por el 55\% está más bien de acuerdo y un 5\% no tiene una opinión definida.

El alumnado destaca el medio RV como una herramienta interesante en el desarrollo del proyecto. Las expectativas quedan cubiertas en un alto porcentaje [punto b) fig. 7], al 9\% totalmente de acuerdo, $67 \%$ más bien de acuerdo y $24 \%$ que no se posiciona. Se destaca que la RV ofrece una aportación distintiva con respecto a otras herramientas tecnológicas, el 47\% totalmente de acuerdo, y el 53\% más bien de acuerdo [punto a) fig. 6].

En relación a la posibilidad de usar la tecnología en el futuro [punto c) fig. 6], el 40\% está totalmente de acuerdo en que si tuviera la opción estaría interesado en su uso, el 55\% más bien de acuerdo y solo el 5\% no se posiciona.

Es mencionable que aquellos alumnos que no habían tenido ninguna experiencia previa con la tecnología destacan que ofrece una visión distintiva con respecto a otras herramientas, por encima de la media del grupo de alumnos que sí había tenido un contacto previo con la tecnología, pese a que el rango de valoración es igualmente alto (tabla 4). Lo mismo sucede 
con la valoración global, que también es superior en aquellos alumnos que utilizan la herramienta por primera vez.

Sin embargo, la valoración de los alumnos que ya conocían la herramienta es más alta en cuestiones como el cumplimiento de sus expectativas, su intención de repetir la experiencia en el futuro y el grado de dificultad en la preparación de los ficheros (tabla 4).

Tabla 4. Resultados del análisis con escala Likert de 5 niveles, en relación a la experiencia previa del alumno con herramientas de RV.

\begin{tabular}{|c|c|c|c|c|c|c|}
\hline & $\begin{array}{c}\text { Experiencia } \\
\text { previa RV }\end{array}$ & $\mathrm{N}$ & Media & $\begin{array}{l}\text { Deviación } \\
\text { Std. }\end{array}$ & Mín. & Máx. \\
\hline \multirow{3}{*}{$\begin{array}{l}\text { Consideras que la herramienta ofrece } \\
\text { una aportación distintiva con respecto a } \\
\text { otras herramientas }\end{array}$} & no & 16 & 4,50 & ,516 & 4 & 5 \\
\hline & si & 4 & 4,25 & ,500 & 4 & 5 \\
\hline & Total & 20 & 4,45 & ,510 & 4 & 5 \\
\hline \multirow{3}{*}{$\begin{array}{l}\text { Opinión global: En su conjunto } \\
\text { considero el uso de la CAVE como una } \\
\text { herramienta interesante en el desarrollo } \\
\text { de proyecto }\end{array}$} & no & 16 & 4,38 & ,619 & 3 & 5 \\
\hline & si & 4 & 4,25 & ,500 & 4 & 5 \\
\hline & Total & 20 & 4,35 & ,587 & 3 & 5 \\
\hline \multirow{3}{*}{$\begin{array}{l}\text { Si tuvieses la opción, te gustaría hacer } \\
\text { uso de la CAVE en futuras ocasiones }\end{array}$} & no & 16 & 4,31 & ,602 & 3 & 5 \\
\hline & si & 4 & 4,50 & ,577 & 4 & 5 \\
\hline & Total & 20 & 4,35 & ,587 & 3 & 5 \\
\hline \multirow{3}{*}{$\begin{array}{l}\text { ¿La experiencia ha cubierto tus } \\
\text { expectativas? }\end{array}$} & no & 16 & 3,69 & ,479 & 3 & 4 \\
\hline & si & 4 & 4,25 & ,500 & 4 & 5 \\
\hline & Total & 20 & 3,80 & ,523 & 3 & 5 \\
\hline \multirow{3}{*}{$\begin{array}{l}\text { Para la visualización del proyecto en un } \\
\text { entorno de realidad inmersiva es } \\
\text { necesario la preparación previa mediante } \\
\text { la aplicación CAVE Preview. Valora la } \\
\text { facilidad de manejo de esta aplicación. } \\
\text { Usabilidad }\end{array}$} & no & 16 & 3,13 & ,806 & 2 & 5 \\
\hline & si & 4 & 3,25 & ,500 & 3 & 4 \\
\hline & Total & 20 & 3,15 & ,745 & 2 & 5 \\
\hline
\end{tabular}

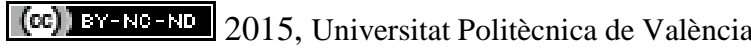

Congreso In-Red (2015) 


\section{Conclusiones}

En relación a los objetivos educativos de la taxonomía de Bloom, la utilización de la RV proporciona la capacidad de desarrollar los niveles más altos de la taxonomía: análisis, síntesis y evaluación. Los dos primeros se establecen en relación a cómo las formas y materiales proyectados se interrelacionan y configuran el conjunto de la instalación. En el proceso se destaca el nivel de evaluación que, recogiendo a los anteriores, es el que permite al alumno contrastar y validar la dimensión, escala, integración con el entorno, apariencia, etc., de las ideas del proyecto en curso.

La implementación de esta herramienta permite una experiencia dinámica y vivencial. El movimiento se vuelve relevante como parte de la representación. Desde una representación inmersiva, el alumno se desplaza e interacciona con el proyecto en un entorno dinámico en el que los movimientos y cambios se efectúan en tiempo real. En este caso, se constata que el uso de la RV como expresión gráfica y herramienta en el desarrollo de proyecto permite de manera vivencial analizar y verificar las ideas trabajadas en el proyecto.

Además, la RV, trascendiendo su aliciente tecnológico y papel instrumental, se destaca tanto como un medio innovador, como una potencial herramienta de comunicación.

La implementación de la herramienta permite, con la incorporación de la dimensión temporal, nuevas formas y métodos de visualización. A diferencia de cualquier otro instrumento, la RV incorpora al desarrollo del proyecto las consideraciones de percepción, tanto la de la propia instalación del proyecto, como la de la integración de la misma con el ámbito de actuación propuesto.

La incorporación del proyecto en un entorno virtual permite que la componente comunicativa de su representación adquiera un importante papel. La experiencia individual para el autor del proyecto se hace extensiva al resto de sus compañeros. El espacio simulado permite de una manera fácil proporcionar una visión holística del conjunto del proyecto sin necesidad de un conocimiento en profundidad. Inmerso en su proyecto, el alumno es capaz de transmitir sus ideas con mayor convencimiento.

Quiero agradecer la colaboración en todo este largo recorrido al Área de Sistemas de Información y Comunicaciones (ASIC), y por extensión al Vicerrectorado para el Desarrollo de las Tecnologías de la Información y de las Comunicaciones de la Universitat Politècnica de València, que han puesto al alcance de toda la comunidad universitaria servicios de tal calidad. 


\section{Referencias}

BELL John T., SCOTT H. (1995) "The Investigation and Application of Virtual Reality as an Educational Tool” Proceedings of the American Society for Engineering Education. Anaheim, California.

HERNANDEZ L., TAIBO J., SEOANE A., JASPE A. (2011) "La percepción del espacio en la visualización de arquitectura mediante realidad virtual inmersiva / Space perception in architectural visualization thought immersive virtual reality” EGA: revista de expresión gráfica arquitectónica n'18, p.252-261

PUYUELO CAZORLA M., VAL FIEL M., MERINO SANJUAN L., FELIP MIRALLES F. (2011) "Representaciones virtuales y otros recursos técnicos en la accesibilidad al patrimonio cultural / Virtual representations and other technical resources for access to cultural Heritage” en EGA: revista de expresión gráfica arquitectónica nº17, p.164-173.

VAL FIEL M., MERINO SANJUAN L, PUYUELO CAZORLA M. (2010) "La expresión gráfica digital: de la representación gráfica a la experiencia inmersiva”. Actas del Congreso Internacional de Expresión Gráfica Arquitectónica XIII. Valencia: DEGA

WINN, W. (1993) “A conceptual basis for educational applications of virtual reality”. Human Interface Technology Laboratory of the Washington Technology Center, University of Washington. Estados Unidos 